V. G. und G. Ghrorro, Helv. med. Acta 25, 235 (1958). - 13. Za Jicek, J. und N. Datta, Acta haemat. 9, 115 (1953). - 14. PILZ, W. und H. HörLEIN, Hoppe-Seyler's Z. physiol. Chem. 339, 157 (1964). - 15. GlrCK, D., Biochem. J. 31, 521 (1937); J. Biol. Chem. 130, 527 (1939). - 16. Alles, G. A. and R. C. Hawes, J. Biol. Chem. 113, 375 (1940). - 17. Mendel, B. and H. Rudney, Biochem. J. 37, 59 (1943). - 18. BrauER, W. R. and M. A. Root, Amer. J. Physiol. 149, 611 (1947). - 19. KaLow, W., Arch. exp. Path. Pharmakol. 215, 370 (1952). - 20. Koelle, G. B., Klin. Wschr. 36, 1043 (1958). - 21. Koelle, G. B. (editor), Cholinesterase and anticholinesterase agents. Handbuch der exper. Pharmakologie Bd. XV. Springer Verlag, Berlin-GöttingenHeidelberg 1963. - 22. Antopol, W., A. Schiprin und L. TucharanN, Proc. Soc. exp. Biol. (N. Y.) 36, 46 (1937). - 23. FremontSmith, K., W. Voliviles und P. A. Wood, J. Lab. a. Clin. Med. 40, 692 (1952). - 24. Vorhaus, L. J., H. H. Scudamore und R. M. KARK, Am. J. M. Sc. 221, 140 (1951). - 25. Vorhaus, L. J. und R. M. KarK, Am. J. Med. 14, 707 (1953). - 26. LaMotta, R. V., H. M. Willians und H. J. Wetstone, Gastroenterology 33, 50 (1957). - 27. LANG, W. und G. INTSESuloguv, Klin. Wschr. 40, 312 (1962). - 28. McCane, R. A., Proc. Roy. Soc. Med. 43, 272 (1950). -29. RenNhold, J. G., L. G. TourignY, und V. L. Yonan, Am. J. Clin. Pathol. 23, 645 (1953). - 30. Schmidr, H. W., Klin. Wschr. 25, 818 (1947). - 31. Cline, J. K., R. B. Johnson und W. H. Johnson, Southern Med. J. 41, 374 (1948). - 32. WILLIAMs, H. M., R. V. LaMotta und H. J. Wetstone, Gastroenterology 33, 58 (1957). - 33. Haminer, O., Ärztl. Forschg. 15, 381 (1961). - 34. Scudamore, H. H., L. J. Vorhaus II, und R. M. Kark, J. Lab. Clin. Med. 37, 860 (1951). - 35. Heinecker, R. und I. MAYer, Klin. Wschr. 95, 340 (1957). - 36. Ammon, R. und F. J. Zapp, Klin. Wschr. 33, 759 (1955). - 37. KaLow, W. und D. R. GunN, Pharmakol. a. Exper. Ther. 120, 203 (1957). - 38. KALow, W. und K. Genest, Canad. J. Biochem. a. Physiol. 35, 339 (1957). - 39. GrohmanN, W., Anaesthesist 6, 136 (1957). - 40. Harris, H. und E. B. Robson, Lancet (Lo.) 218 (1963). - 41. Ammon, R. und K. ZIPF, Klin. Wschr. 20, 1177 (1941). - 42. Heim, F. und D. AMELUNG, Arch. exper. Path. Pharmakol. 207, 477 (1949). - 43. ZIPF, H. F., in: Killian, H. (editor): Lokalanästhesie und Lokalanästhetika, Georg Thieme Verlag, Stuttgart 1959. - 44. FREUDENBERG, R. und F. K. Redicich, Arch. exp. Path. Pharmak. 188, 645 (1938). - 45. Moore, C. B., R. Birchall, H. M. HoracK und H. M. Batson, Am. J. Med. Sci. 234, 538 (1957). - 46. Grob, D.,
Ch. 23 in Koelle, G. B. (editor): Handbuch der exper. Pharmakologie Bd. XV., Springer Verlag 1963. - 47. MüLLER, P. (editor), DDT, Vol. I (1955), Vol. II (1959) Birkhäuser Verlag, Basel-Stuttgart. - 48. Schrader, G:, Die Entwicklung neuer insektizider Phosphorsäure-Ester. Verlag Chemie G. M. B. H., Weinheim 1963. -49. Wrrth, W., Arch. exp. Path. Pharmak. 207, 547 (1949); 217, 144 (1953). - 50. Hechr, G. und W. Wrrth, Arch. exp. Path. Pharmak. 211, 264 (1950). - 51. Holmstedt, B., in Koelle, G. B. (editor), vgl. 21. - 52. Wirson, I. B., in: The Enzymes, Vol. IV., 501 (1960), Academic Press, N. Y.-Lo. 1960. - 53. Metcalf, R. L., J. Econ. Entomol. 44, 883 (1951). Organic Insecticides. N. Y. London, Inc. Publishers 1955. - 54. Frawley, J. P., E. C. HAGAN und O. G. Firzhugh, J. Pharmakol. Exp. Therap. 105, 156 (1952). - 55. Hambin, D. O. und H. F. Marchand, Cholinesterase tests and their applicability in the field. N.Y., American Cyanamid Company, March 1951; Hamblin, D. O. und H. F. Marchand, Ann. Int. Med. 36, 50 (1952). - 56. НECHT, G., Dtsch. med. Wschr. 77, 783 (1952). - 57. Pribilla, O., Arch. f. Toxik. 15, 210 (1955). - 58. ErdmanN, W. D. und L. Lendle, Ergeb. inn. Med. Kinderheilk. 10, 104 (1958). - 59. Durfam, W. F. und W. J. Hayes, Jr., Arch. environm. Hlth. 5, 21 (1962). - 60. AcKermanN, H. Dtsch. Gesd. wes. 26, 1213 (1964). - 61. KLIMMER, O. R., Pflanzenschutz und Schädlingsbekämpfungsmittel. Hundt-Verlag, Hattingen (Ruhr), 1964. - 62. Wotr, H., Klin. Wschr. 43, 819 (1965). 63. BRECht, K., Dtsch. med. Wschr. 72, 445 (1947). - 64. BARNES, J. M. und D. R. Davies, Brit. Med. J. 2, 316 (1951). - 65. Callaway, S., D. R. Davies und J. P. Rutland, Brit. Med. J. 2, 812 (1951). - 66. Marchand, J. F., J. Am. Med. Assoc. 149, 738 (1952). - 67. Wolfsie, J. H. und G. D. Winter, A. M. A. Arch. Industr. Hyg. 6, 43 (1952). - 68. MEYER, A. und W. WILBRANDT, Helv. Physiol. Acta 12, 206 (1954). - 69. Augustinsson, K.-B., Acta physiol. Scand. 35, 40 (1955). - 70. FRYER, J. H., R. G. D. Steel und H. H. Wriliams, A. M. A. Arch. Ind. Hith. 12, 406 (1955). - 71. SAILER, S. und H. Braunsteiner, Klin. Wschr. 37, 986 (1959). - 72. Shanor, S. P. und Mitarbeiter, Am. J. Med. Sci. 242, 141 (1961). - 73. Augussinsson, K.-B., in: Methods of biochemical analysis, N. Y., Intersc. Publishers, Inc., Vol. 5, 1 (1957). - 74. Wirter, R. F., Arch. environm. Hlth. 6, 537 (1963). - 75. W $\mathrm{IRTH}$, W., unveröffentlicht. - 76. MrcheL, H. O., J. Lab. Clin. Med. 34, 1564 (1949). - 77. Hestrin, S., J. biol. Chem. 180, 249 (1949). - 78. Pirz, W., Klin. Wschr. 43, 1227 (1965). - 79. GANELIN, R. S., Arizona Medicine (1964) 710

Prof. Dr. G. Hecht 2401 Lübeck-Brodten

\title{
Dünnschichtchromatographischer Nachweis von Prednisolon-21-Hemisuccinat-Natrium und seiner Metaboliten
}

\author{
Von F. Weist, L. Zicha und Ch. Schöttrer
}

\section{Aus der Mediqinischen Universitätsklinik Erlangen (ebcm. Direktor: Prof. Dr. Dr. b. c. N. Henning) und dem Städtiscben Krankenbaus Pegnitz (Chefarzt: OMR Dr. W. Mauelshagen)}

(Eingegangen am 30. Juli 1966)

Es wird über die Variation einer dünnschichtchromatographischen Methode zur Auftrennung und zum semiquantitativen Nachweis eines Steroidgemisches von Prednisolon, Prednisolon-21-hemisuccinat-Natrium und Prednisolon-Acetat berichtet. Die Art der Methode gestattet es, zu einem quantitativen Bestimmungsverfahren ausgebaut zu werden.

A modified, thin layer chromatographic separation and semi-quantitative detection is reported for a steroid mixture, consisting of prednisolone, sodium prednisolone-21-hemisuccinate and prednisolone acetate. This type of method can be converted into a quantitative determination.

Bei der Bearbeitung von zahlreichen Problemen des Stoffwechsels synthetischer Glucocorticoidderivate hat sich die Dünnschichtchromatographie bestens bewährt
(1-4). Zwar wurde dieses spezielle Gebiet von uns früher bereits bearbeitet $(2,4)$, doch konnten wir nun durch Änderung der Versuchsbedingungen eine weiter- 
Tab. 1 Untersuchte Fließmittelsysteme

\begin{tabular}{|c|c|c|c|c|}
\hline & Fließmittelsystem & $\begin{array}{l}\text { Prednisolon- } \\
\mathrm{C}_{n,}-\mathrm{OH}\end{array}$ & $\begin{array}{l}R_{F}-\text { Werte von } \\
\text { Prednisolon- } \\
\text { acetat }\end{array}$ & $\begin{array}{l}\text { Prednisolon-21- } \\
\text { Hemisuccinat- } \mathrm{Na}\end{array}$ \\
\hline & Einfache Entwicklung & & & \\
\hline $\begin{array}{l}\text { A } \\
\text { B } \\
\text { C } \\
\text { D }\end{array}$ & Zweifache Entwicklung & & & \\
\hline \multirow{2}{*}{$\begin{array}{l}E \\
F \\
G\end{array}$} & 1. Fließmittel: D & 0,5 & 0,75 & 0,6 \\
\hline & $\begin{array}{l}\text { 1. Fließmittel: Methanol: Tetrachlorkohlenstoff }=15: 85 \\
\text { 2. Fließmittel: Chloroform : Diäthyläther: Eisessig }=70: 20: 10\end{array}$ & 0,4 & 0,7 & 0,42 \\
\hline
\end{tabular}

Tab. 2 Farbtönungen von Prednisolon- $\mathrm{C}_{21}-\mathrm{OH}$, Prednisolon- $\mathrm{C}_{21}$-acetat und Prednisolon-21-hemisuccinat-Natrium bei verschiedenen Farbreagenzien

\begin{tabular}{|c|c|c|c|c|}
\hline Farbreagenzien & Anfärbung Trocknung & Tageslicht & $\mathrm{UV} ; 365 \mathrm{~m} \mu$ & UV; $254 \mathrm{~m} / \mathrm{u}$ \\
\hline $\begin{array}{l}\text { Perchlorsäure, 20-proz. } \\
\text { Perchlorsäure, 20-proz. } \\
\text { Zink-II-chlorid, ges. Lsg. in Eisessig } \\
\text { Orthop hosphorsäure, 40-proz. } \\
\text { Cer-IV-sulfat-Schwefelsäure (8) } \\
\text { Jodreagenz-1) }\end{array}$ & $\begin{array}{l}10 \mathrm{Min} \text {. bei } 100^{\circ} \\
20 \mathrm{Min} \text {. bei } 130-140^{\circ} \\
\text { föhngetrocknet }\end{array}$ & $\begin{array}{c}\text { beige } \\
\text { dunkelbraun } \\
\text { beige } \\
\text { braun } \\
\text { dunkelbraun } \\
\text { dunkelbraun }\end{array}$ & $\begin{array}{l}\text { gelb } \\
\text { dunkelbraun } \\
\text { graugelb } \\
\text { gelbgrün } \\
\text { orange }\end{array}$ & $\begin{array}{l}\text { braun } \\
\text { schwarz } \\
\text { beige } \\
\text { grünbraun } \\
\text { rostbraun }\end{array}$ \\
\hline
\end{tabular}

1) Lsg. I : $8 \mathrm{~g}$ Kaliumiodid und $4 \mathrm{~g}$ Jod gelöst in etwa $65 \mathrm{ml}$ 90-proz. Methanol; Lsg. II : 38-proz. Salzsäure : Methanol = 2 : 3 ; $1 \mathrm{TI}$. Lsg. I wird mit 2 Tin. Lsg. II gemischt, filtriert und als Sprühreagenz verwendet.

gehende Differenzierung einzelner dünnschichtchromatographisch nachweisbarer Stoffwechselprodukte erzielen. Die Verwendung einer Kombination verschiedener Fließmittelsysteme erlaubte schließlich die pharmakochemische Prüfung der Fragestellung, warum trotz Applikation höchster Glucocorticoid-Dosen manche Patienten keine Nebenwirkungen im Sinne des CusbingSyndroms aufweisen. Es hat sich hierbei gezeigt, daß dies nicht zuletzt eine Frage der unterschiedlichen Metabolisierung von synthetischen Steroiden ist $(6,7)$. Im Rahmen dieser methodischen Arbeit soll einewsemiquantitative Bestimmungsmethode für Prednisolon-21hemisuccinat-Natrium ${ }^{1}$ ) bzw. dessen Metaboliten beschrieben werden, die sich auch für die quantitative Bestimmung dieser Substanzen eignet.

\section{Methodik und Ergebnisse}

Wie bei früheren Untersuchungen arbeiteten wir auch hier nach der Originalmethode von Stafl $(3,5)$. Es kamen $20 \times 20 \mathrm{~cm}$ DC-Platten, beschichtet mit Kieselgel $G$ in einer Dicke von ungefähr $250 \mu$ zur Anwendung. Die Laufstrecke betrug $15 \mathrm{~cm}$. Es wurden je ein Teil Urin, Duodenalflüssigkeit und Serum mit 3 Teilen 1,2Dichloräthan $10 \mathrm{Min}$. geschüttelt, die beiden Phasen im Scheidetrichter getrennt und dieser Vorgang in gleicher Weise noch zweimal wiederholt. Die gesammelten Extraktmengen wurden bei $40^{\circ}$ im Vakuum zur Trockene eingeengt. Auf eine vorherige Reinigung des Extraktes, etwa durch Ausschüutteln mit $0,1 \mathrm{~N} \mathrm{NaOH}$, konnte zumindest bei der Urinextraktion verzichtet werden. Der trockene Rückstand wurde in $0,5 \mathrm{ml}$ Chloroform gelöst.

Gegenüber der früher geübten Extraktionsmethóde mit Chloroform, Diäthyläther oder Butanol konnte durch Verwendung von 1,2-Dichloräthan die quantitative Ausbeute wesentlich verbessert

1) „Solu-Decortin-H“ der Fa. E. Merck AG., Darmstadt. werden. Außerdem war bei Verwendung von 1,2-Dichloräthan die Neigung zur Emulsionsbildung weniger stark ausgeprägt als bei Chloroform. Schließlich ließ sich auch der zeitliche Aufwand bei der Vakuumeinengung, insbesondere gegenüber dem Butanolextrakt, stark reduzieren. Bei Diäthyläther als Extraktionsmittel wāren die Verhältnisse in dieser Hinsicht zwar noch günstiger, doch ist die Phasentrennung mit Hilfe des Scheidetrichters umständlicher, da die spezifische Dichte von Diäthyläther kleiner als die von biologischen Flüssigkeiten ist. Es muß allerdings bemerkt werden, da $\beta$ die Wiedergewinnungsrate für manche Metaboliten im Diäthylätherextrakt günstiger war, jedoch wurden auch vermehrt störende Begleitstoffe im Extrakt aufgenommen.

Neben den Reinsubstanzen Prednisolon- $\mathrm{C}_{21}-\mathrm{OH}$, Prednisolon-21-hemisuccinat-Natrium und Prednisolon- $\mathrm{C}_{21}$ acetat $^{2}$ ) wurden die Extrakte in steigender Menge mittels Blutzuckerpipetten auf DC-Platten aufgetragen. Die Trennung erfolgte bei aufsteigender Technik in gesättigter Atmosphäre, die semiquantitative Auswertung nach Anfärbung im sichtbaren und filtrierten UV-Licht (3).

Von einer größeren Anżahl von Fließmittelgemischen erwiesen sich die in Tabelle 1 zusammengestellten als brauchbar. Das Fließmittel F fand bevorzugt Anwendung. Die Farbreaktion erfolgte mit Cer(IV)-sulfatSchwefelsäure-Reagenz (8), das gewisse Vorteile gegenüber anderen Farbreagenzien besitzt. So können im filtrierten UV-Licht bei $365 \mathrm{~m} \mu$ noch unter $0,1 \mu \mathrm{g}$ der Prednisolonverbindungen als Reinsubstanz nachgewiesen werden. Die Anfärbung der Fraktionen bei Verwendung verschiedener Farbreagenzien sind der Tabelle 2, die Zeitabhängigkeit der Harnkonzentration der einzelnen Substanzen ist der Abbildung $1 \mathrm{zu}$ entnehmen. Das Chromatogramm der Abbildung 2 zeigt die Auftrennung einzelner Extrakte von fraktioniert gesammeltem Urin sowie die 3 Reinsubstanzen Prednisolon- $\mathrm{C}_{21}-\mathrm{OH}$, Prednisolon- $\mathrm{C}_{21}$-acetat und Prednisolon-21hemisuccinat-Natrium.

2) Wir danken der Fa. E. Merck AG. für die freundliche Úberlassung von Reinsubstanzen. 


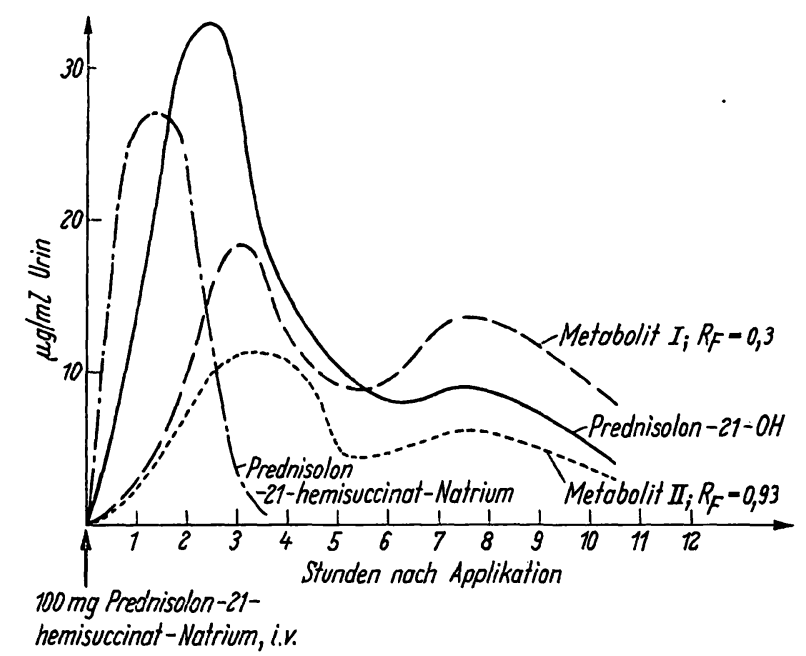

Abb. 1

Ausscheidung von Prednisolon-21-hemisuccinat-Natrium und seiner Metaboliten im Harn nach intravenöser Applikation

\section{Diskussion}

Gegenüber den früher angewandten dünnschichtchromatographischen Trennungs- und Nachweisverfahren ergaben sich einige wesentliche Verbesserungen. So hat 1,2-Dichloräthan als Extraktionsmittel gegenüber Chloroform nicht allein den Vorteil, daß die Wiedergewinnungsrate für die untersuchten Substanzen verbessert werden konnte, sondern es ließ sich auch der zeitliche Aufwand bei der Extraktion wesentlich verringern. Durch die Wahl der Cer(IV)-sulfat-Schwefelsäure-Anfärbung war auch gesichert, daß die DC-Platten bei konstantbleibender Intensität der Farbreaktion über Monate aufbewahrt werden können, was wegen der hygroskopischen Reaktion nach einer Phosphorsäurebehandlung der DC-Platten nicht möglich ist. Der wesentliche Fortschritt des nun angewandten Fließmittels besteht darin, daß auch für Prednisolon-21-hemisuccinat-Natrium ein günstiger $R_{\mathrm{F}}$-Wert erzielt und die 3 in Frage kommenden Substanzen weiter voneinander getrennt werden konnten. Bei dem Fließmittelsystem C lagen die $R_{\mathrm{F}}$-Werte der einzelnen Substanzen zwar noch günstiger als bei dem System F, doch war ihre Abgrenzung gegenüber Fraktionen, die normalerweise im Urin vorkommenden Komponenten entsprechen, weniger gut möglich.

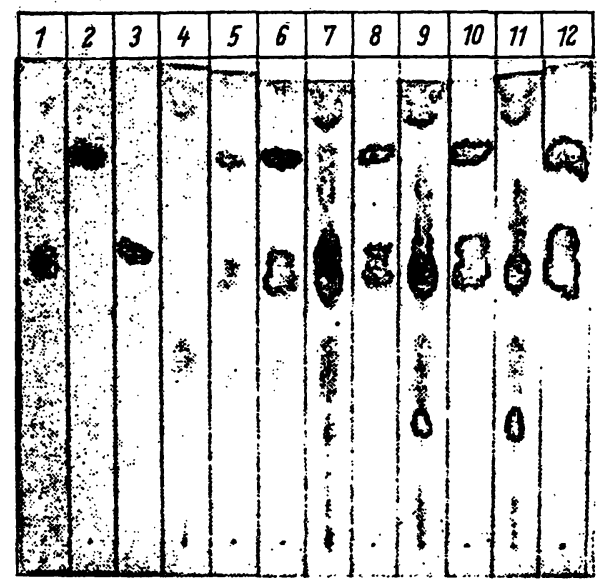

Abb. 2

Chromatogramm

Spalte 1: $30 \mu \mathrm{g}$ Prednisolon $-\mathrm{C}_{21}-\mathrm{OH}$

Spalte 2: $30 \mu \mathrm{g}$ Prednisolon- $\mathrm{C}_{21}$-acetat

Spalte 3: $30 \mu \mathrm{g}$ Prednisolon-21-hemisuccinat-Natrium

Spalte 4: Urinleerwertextrakt

Spalte 5: $10 \mu \mathrm{g}$ der drei 0 . g. Pred.-Verbindg.

Spalte 6: Urinleerwertextrakt $+30 \mu \mathrm{g}$ der drei o. g. Pred.-Verbindg.

Spalte 7: Urinextrakt (Sammelportion 1. Std.)

Spalte 8: $30 \mu \mathrm{g}$ der drei 0. g. Pred.-Verbindg.

Spalte 9: Urinextrakt (Sammelportion 2. Std.)

Spalte 10: $50 \mu \mathrm{g}$ der drei o. g. Pred.-Verbindg.

Spalte 11: Urinextrakt (Sammelportion 3. Std.)

Spalte 12: $100 \mu \mathrm{g}$ der drei 0. g. Pred.-Verbindg.

In der Sammelportion der 1. Std. (Spalte 7) sind von unten nach oben : der Metabolit I; eine auch im Leerwert vorkommende Fraktion Prednisolon- $\mathrm{C}_{21}-\mathrm{OH}$; Prednisolon-21-hemisuccinat-Natrium; einige auch im Leerwert vorkommende Komponenten und der Metabolit II $z u$ erkennen.

In der Sammelportion der 2. und 3. Std. (Spalte 9 und 11) nimmt die Konzentration des Metabolit $I$, des Prednisolon- $\mathrm{C}_{21}-\mathrm{OH}$ sowie des Metabolit II zu, während Prednisolon-21-hemisuccinat-Natrium in der 3. Std. nur noch in äußerst geringer Konzentration nachzuweisen ist. (Vgl. auch Abb. 1.)

Mit dieser Methode gelang es, bei Patienten, die Prednisolon-21-hemisuccinat-Natrium in einer Dosierung bis $100 \mathrm{mg}$ i. v. appliziert bekamen, sowohl dir injizierte Substanz, den $C_{21}$-freien-Alkohol, als auch 6 weitere, im Leerwert nicht auftretende Fraktionen, nachzuweisen. Eine dieser Fraktionen verhält sich in der Anfärbung und nach den $R_{\mathrm{F}^{-}}$-Werten wie das $\mathrm{C}_{21}$-Acetat des Prednisolons. Bei Anwendung einer entsprechenden Farbreaktion ist es schließlich möglich, nach Abkratzen der verschiedenen Substanzen und Eluierung aus dem Kieselgel, diese photometrisch zu bestimmen.

\section{Literatur}

1. FunK, F.-W. und L. ZichA, Med. Exp. 7, 1 (1962). - 2. SChEIFFARTh, F., L. Zicha, W.-F. Funck und M. Engelhardt, Acta endocrin. (K'hचn) 43, 227 (1963). - 3. STAHL, E., Dünnschichtchromatographie, Springer Verlag Berlin-Göttingen-Heidelberg (1962). - 4. Zicha, L., F. ScheIfFarth, D. BergNer und U. Eyselein, Acta endocrin. (K'hvn) Suppl. 67, 94 (1962). - 5. STAHL, E., Chemikerzeitung 82, 323 (1958). - 6. WEBER, G. und
Mitarbeiter, persönl. Mitteilung. - 7. Werst, F., L. ZrChA, G. WeBer und $\mathrm{CH}_{\mathrm{H}}$. SchötrNer, Untersuchungen über verminderte Nebenwirkungen einer Glucocorticoidtherapie bei best. dermatol. Erkrankungen, Endokrinologie (in Vorbereitung). - 8. „Anfärbereagenzien für Dünnschicht- und Papierchromatographiec E. Merck AG Darmstadt.
Dr. F. R. Weist 2400 Lübeck

Kronsforder Allee $71-73$ 